\title{
高效液相色谱-串联质谱法检测牛奶中头孢洛宁残留
}

\author{
李帅鹏 ${ }^{1}$ ， 郭春娜 ${ }^{1}$ ， 孟 蕾 $^{2}$, 黄显会 ${ }^{*}$
}

(1. 华南农业大学国家兽药残留基准实验室, 广东 广州 510642; 2. 河南省兽药监察所, 河南 郑州 450008)

摘要: 建立了牛奶中头孢洛宁残留检测的高效液相色谱-串联质谱方法。1 $\mathrm{g}$ 牛奶经乙腈沉淀蛋白质后, 上清液于 $37{ }^{\circ} \mathrm{C}$ 水浴下氮气吹干, 用 $1 \mathrm{~mL}$ 甲醇- $0.1 \%$ 甲酸水溶液 $(3: 7, \mathrm{v} / \mathrm{v})$ 复溶, 正己烷除脂净化后检测。流动相为乙腈和 $0.1 \%$ 甲酸水溶液, 梯度洗脱, 经 $\mathrm{C}_{18}$ 色谱柱分离, 采用多反应监测正离子模式对头狍洛宁进行定性定量分析。采用 基质匹配法对牛奶中头孢洛宁的含量进行标准校正, 在 $2 \sim 200 \mu \mathrm{g} / \mathrm{L}$ 范围内, 头孢洛宁质量浓度与其对应峰面积的 线性关系良好, 相关系数>0.999。牛奶中加标样品的检出限 (按 $S / N \geqslant 3$ 计) 为 $0.5 \mu \mathrm{g} / \mathrm{kg}$, 定量限 ( $S / N \geqslant 10$ 计) 为 $2 \mu \mathrm{g} / \mathrm{kg}$ 。在定量限、 $1 / 2$ 最高残留限量、最高残留限量、 2 倍最高残留限量添加水平下, 牛奶中头孢洛宁的平均回 收率为 $78.5 \% \sim 86.2 \%$, 日内相对标准偏差为 $1.5 \% \sim 6.2 \%$, 日间相对标准偏差为 $2.9 \% \sim 5.6 \%$ 。该方法可用于牛奶 中头孢洛宁的残留检测。

关键词:高效液相色谱; 串联质谱; 头孢洛宁; 牛奶; 残留

中图分类号: O658 文献标识码:A 文章编号: 1000-8713(2014)05-0519-05

\section{Determination of cefalonium residue in milk by high performance liquid chromatography-tandem mass spectrometry}

\author{
LI Shuaipeng ${ }^{1}$, GUO Chunna ${ }^{1}$, MENG Lei ${ }^{2}$, HUANG Xianhui ${ }^{1 *}$ \\ (1. National Reference Laboratory of Veterinary Drug Residues, South China Agricultural University, \\ Guangzhou 510642, China; 2. Henan Institute of Veterinary Drug Control, Zhengzhou 450008, China)
}

Abstract: An analytical method was developed for the determination of cefalonium in milk by high performance liquid chromatography-tandem mass spectrometry (HPLC-MS/MS). A total of $1 \mathrm{~g}$ milk was deproteinized by acetonitrile. The supernatant was transferred into a test tube to be blown dry with $\mathrm{N}_{2}$ at $37{ }^{\circ} \mathrm{C}$. Then the residue was dissolved with methanol-0. $1 \%$ formic acid in water $(3: 7, \mathrm{v} / \mathrm{v})$. The sample was determined by HPLC-MS/MS after the purification. The chromatographic separation was achieved on a $\mathrm{C}_{18}$ column using $0.1 \%$ formic acid in water and acetonitrile as mobile phases with gradient elution. Qualitative and quantitative analyses were achieved by HPLC-MS/MS under positive ionization and multiple reaction monitoring (MRM) mode. Matrix-matched calibration curve was used for the quantification. Good correlation coefficients were obtained $(r>0.999)$ in the mass concentration range of $2-200 \mu \mathrm{g} / \mathrm{L}$. The limit of detection ( $\mathrm{LOD}, S / N \geqslant 3$ ) was $0.5 \mu \mathrm{g} / \mathrm{kg}$ in milk, and the limit of quantification (LOQ, $S / N \geqslant$ 10) was $2 \mu \mathrm{g} / \mathrm{kg}$. The mean recoveries at the four levels of LOQ, 1/2MRL (maximum residue level), MRL, 2MRL were between 78. 5\% and 86. 2\%, with the intra-day relative standard deviations (RSDs) of $1.5 \%$ to $6.2 \%$ and inter-day RSDs of $2.9 \%$ to $5.6 \%$. In conclusion, the established method can be applied for the determination of cefalonium residue in milk.

Key words : high performance liquid chromatography (HPLC); tandem mass spectrometry ( MS/MS) ; cefalonium; milk; residue

头孢洛宁 ( cefalonium) 是美国先灵葆雅 (Schering-Plough) 制药公司在英联邦国家内开发
的奶牛枯奶期针对乳房炎的预防性用药, 是全球 3 个指定的动物专用头孢类抗生素之一, 属于第二代 
头孢类抗生素 ${ }^{[1]}$ 。头孢洛宁对酸和 $\beta$-内酰胺酶稳 定, 杀菌力强, 抗菌谱广, 对革兰氏阴性菌和革兰氏 阳性菌均有效; 尤其对引起奶牛乳房炎的大多数病 原菌有效,主要用于细菌感染引起的奶牛乳房炎的 防治 ${ }^{[2-4]}$ 。目前该药只在日本、新西兰、澳大利亚、 欧盟和英联邦国家范围内使用,美国、加拿大等市场 未使用该药,我国也未见进口或开发报道。

头狍菌素类抗生素的过量使用具有毒副作用， 主要体现在对脑神经、听觉以及肾脏的损害 ${ }^{[5]}$, 对 人体健康造成危害。因此, 世界各国对动物源性食 品中的头孢洛宁残留量均提出了限量要求, 欧盟规 定牛奶中头孢洛宁的最高残留限量 (MRL) 为 10 $\mu \mathrm{g} / \mathrm{kg}^{[6]}$, 日本肯定列表规定牛组织和牛奶中头孢 洛宁的最高残留限量均为 $10 \mu \mathrm{g} / \mathrm{kg}^{[7]}$ 。

国外头孢洛宁乳房注人剂 (Cepravin Dry Cow $250 \mathrm{mg}$ Intramammary suspension (Dry Cow)) 已 上市使用多年,但有关牛奶中头孢洛宁残留检测方 法的报道较少, 主要有微生物法 ${ }^{[8]}$ 、酶联免疫分析 法 $^{[9,10]}$ 和高效液相色谱-串联质谱法 ${ }^{[11,12]}$ 。牛奶中 头孢洛宁的 MRL 为 $10 \mu \mathrm{g} / \mathrm{kg}$, 且牛奶中干扰基质 较多,HPLC 法不能满足残留检测的要求。本研究 采用高效液相色谱-串联质谱法 (HPLC-MS/MS ) 分 析牛奶中头孢洛宁残留,并进行了方法学评价。

\section{1 实验部分}

\section{1 仪器与试剂}

API 4000 电喷雾-串联四极杆质谱仪, 配 Analyst 4. 1.5 软件 (美国应用生物系统公司); Agilent 1200 型液相色谱仪 (美国安捷伦公司)；B-260 型恒 温水浴锅 (上海雅荣生化仪器设备有限公司); Milli-Q 纯水机 (美国 Millipore 公司); Mach1. 6 R 冷冻型离心机 (美国 Thermo 公司)。头狍洛宁标 准物质: 产品编号 32904, CAS 号 5575-21-3, 由美国 Sigma 公司提供; 正己烷为国产分析纯; 甲醇、乙腈、 甲酸均为色谱纯 (美国 Fisher 公司)。

\section{2 溶液的配制}

头孢洛宁标准储备液 $(500 \mathrm{mg} / \mathrm{L})$ : 准确称取头 孢洛宁标准物质 $5.00 \mathrm{mg}$, 置于 $10 \mathrm{~mL}$ 容量瓶中, 用 $30 \%(\mathrm{v} / \mathrm{v})$ 乙腈水溶液溶解并定容至刻度, 配成 500 $\mathrm{mg} / \mathrm{L}$ 标准溶液, 于 $4{ }^{\circ} \mathrm{C}$ 冰箱中避光保存。临用前 用甲醇- $0.1 \%$ 甲酸水溶液 $(3: 7, \mathrm{v} / \mathrm{v})$ 稀释成头孢洛 宁系列标准工作液, 现用现配。0. $1 \%$ 甲酸水溶液: 取 $1 \mathrm{~mL}$ 甲酸,置于 $1000 \mathrm{~mL}$ 容量瓶内, 用超纯水定 容至刻度, 超声后使用。甲醇- $0.1 \%$ 甲酸水溶液 (3: $7, \mathrm{v} / \mathrm{v})$ : 取 $30 \mathrm{~mL}$ 甲醇与 $70 \mathrm{~mL} 0.1 \%$ 甲酸水混匀
即得。

\section{3 样品前处理}

准确称取 $(1.00 \pm 0.01) \mathrm{g}$ 牛奶置于 $15 \mathrm{~mL}$ 离心 管中。加人 $5 \mathrm{~mL}$ 乙腈, 浴旋 $1 \mathrm{~min}$, 置于振荡器上 以 $300 \mathrm{r} / \mathrm{min}$ 振荡 $10 \mathrm{~min}$, 在 $4{ }^{\circ} \mathrm{C}$ 条件下, 以 9000 $\mathrm{r} / \mathrm{min}$ 离心 $10 \mathrm{~min}$ 。转移上清液至 $10 \mathrm{~mL}$ 试管中, 在 $37{ }^{\circ} \mathrm{C}$ 水浴中氮气吹干, 加人 $1 \mathrm{~mL}$ 甲醇- $0.1 \%$ 甲 酸水溶液 $(3: 7, \mathrm{v} / \mathrm{v})$ 复溶, 浴旋 $1 \mathrm{~min}$, 溶液转移至 $2 \mathrm{~mL}$ 离心管中, 加人 $0.8 \mathrm{~mL}$ 正已烷除脂, 浴旋 1 $\min$, 在 $4{ }^{\circ} \mathrm{C}$ 下 $15000 \mathrm{r} / \mathrm{min}$ 离心 $10 \mathrm{~min}$, 弃去正己 烷, 过 $0.22 \mu \mathrm{m}$ 有机相针式过滤器至 $2 \mathrm{~mL}$ 棕色进 样瓶中,LC-MS/MS 分析。

\section{4 色谱-质谱条件}

1.4.1 色谱条件

色谱柱: 美国 Penomenex Luna $\mathrm{C}_{18}(150 \mathrm{~mm} \times$ $2.1 \mathrm{~mm}, 3.5 \mu \mathrm{m})$; 流动相: 乙腈 $(\mathrm{A}), 0.1 \%$ 甲酸水 溶液 $(\mathrm{B})$ 。梯度洗脱程序: 0. $0 \sim 0.5 \mathrm{~min}, 10 \% \mathrm{~B}$; $0.5 \sim 1 \mathrm{~min}, 10 \% \mathrm{~B} \sim 95 \% \mathrm{~B} ; 1 \sim 4 \mathrm{~min}, 95 \% \mathrm{~B}$; $4 \sim$ 4. $5 \mathrm{~min}, 95 \% \mathrm{~B} \sim 10 \% \mathrm{~B} ; 4.5 \sim 10 \mathrm{~min}, 10 \% \mathrm{~B}$ 。流 速: $0.25 \mathrm{~mL} / \mathrm{min}$; 柱温: $35{ }^{\circ} \mathrm{C}$; 进样量: $5 \mu \mathrm{L}$ 。

\subsection{2 质谱条件}

用多反应监测 (MRM) 扫描模式; 电喷雾离子源 (ESI) ; 正离子扫描; 电喷雾电压 $4 \mathrm{kV}$; 离子源温度 $600{ }^{\circ} \mathrm{C}$; 辅助气压力 $35 \mathrm{kPa}$; 雾化气压力 $55 \mathrm{kPa}$; 气

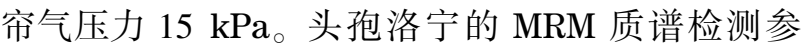
数: 选择离子对 $m / z 459.4 / 337.3,459.4 / 152.3$, 其 中定量离子对为 $m / z 459.4 / 337.3$; 碰撞能量 $(\mathrm{CE})$ $14 \mathrm{eV}$; 去簇电压 $(\mathrm{DP}) 70 \mathrm{~V}$; 碰撞室射出电压 $(\mathrm{CXP})$ $10 \mathrm{~V}$, 射人电压 $(\mathrm{EP}) 10 \mathrm{~V}$ 。

\section{2 结果与讨论}

\section{1 质谱参数的确定}

头孢洛宁标准溶液 $(1 \mathrm{mg} / \mathrm{L})$ 分别在正离子和 负离子模式下全扫描, 发现头孢洛宁在正离子模式 下的响应值更高。头狍洛宁标准溶液 $(1 \mathrm{mg} / \mathrm{L})$ 在 正离子模式下全扫描, 得到 $[\mathrm{M}+\mathrm{H}]^{+}(m / z 459.4)$ 准分子离子峰。对 $m / z 459.4$ 进行二级质谱全扫 描,得到头孢洛宁二级全扫描质谱图 (见图 1)。结 合头孢菌素质子化离子的一般断裂规律 ${ }^{[13]}$ 可知, 头 孢洛宁的质谱行为较简单, 主要为 $\mathrm{R} 2$ 断裂 (见图 2) 得到的 $m / z 123.4$ 和 337.3; C (6)-C (7) 键及 $\mathrm{C}(7)-\mathrm{C}(8)$ 键断裂导致 $\beta$-内酰胺环开环得到的 $m / z$ 152.3 和 309.3 ; 而 $m / z 337.3$ 进一步裂解又可得到 $m / z 152.3$ 。其中 $m / z 337.3$ 和 152.3 的强度相对 较大, 干扰较小, 故选择 $m / z 337.3$ 作为定量离子, 


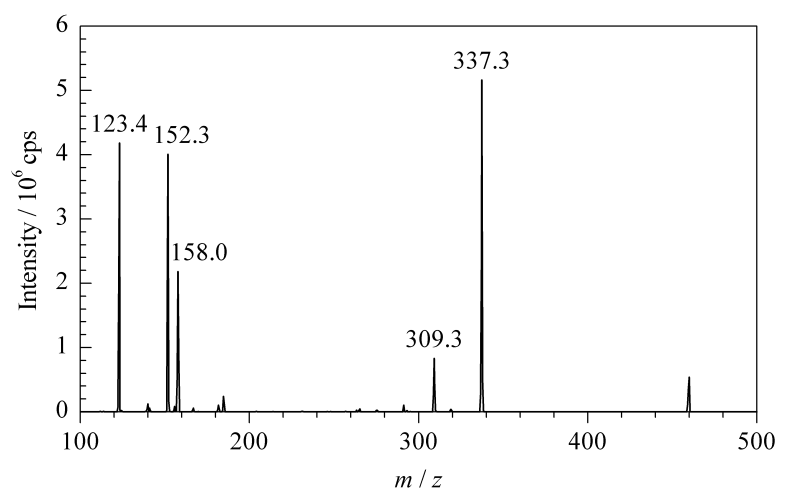

图 1 头孢洛宁的二级质谱图

Fig. 1 MS/MS spectrum of cefalonium

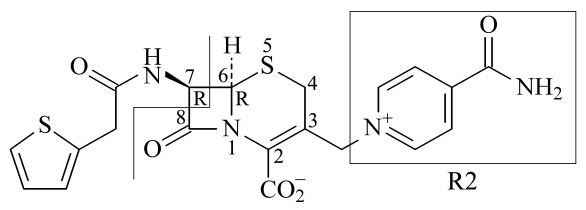

图 2 头狍洛宁的结构式

Fig. 2 Chemical structure of cefalonium

$m / z 152.3$ 为辅助定性离子。

\section{2 色谱条件的优化}

头孢洛宁属于 $\beta$-内酰胺酶类抗生素, 结构式中 既有游离羧基, 又带有氨基, 为两性物质, 极性 小 ${ }^{[14]}$ 。所选色谱柱的填料应为直链烷烃键合的硅 胶, 其中最常见的为十八烷基键合硅胶 (即 $\mathrm{C}_{18}$ )。 研究对比了 Phenomenex Luna $\mathrm{C}_{18}(150 \mathrm{~mm} \times 2.1$ $\mathrm{mm}, 3.5 \mu \mathrm{m})$ 和 Waters $\mathrm{C}_{18}(150 \mathrm{~mm} \times 2.1 \mathrm{~mm}, 5$ $\mu \mathrm{m})$ 色谱柱。两种色谱柱对头孢洛宁均有良好的 保留,头孢洛宁均可得到优异的峰形。综合考虑, 本 文选择 Phenomenex Luna $\mathrm{C}_{18}(150 \mathrm{~mm} \times 2.1 \mathrm{~mm}$, $3.5 \mu \mathrm{m})$ 色谱柱。为了改善峰形及提高分离度,增 强目标物在反相色谱柱上的保留, 经常在流动相溶 液中添加甲酸、乙酸等酸性物质或者甲酸铵、乙酸铵 等缓冲盐。在水相中添加 $0.1 \%$ 的甲酸 ${ }^{[15]}$ 及对流动 相条件的调整能使头孢洛宁在色谱柱上较好的保留 (保留时间在 $6.1 \mathrm{~min}$ 左右), 响应值高, 峰形优良。

\section{3 样品前处理方法的优化}

\subsection{1 样品前处理方法的考察}

有关牛奶中头孢洛宁残留检测方法报道的文献 资料中,有的采用离心的方法预先除去脂肪 ${ }^{[11]}$, 有 的采用乙腈沉淀蛋白 ${ }^{[16]}$, 有的添加 $\mathrm{pH} 8.5$ 磷酸盐 缓冲液稀释牛奶 ${ }^{[12]}$ 然后过固相萃取 (SPE) 柱净化。 文献报道的样品前处理方法中, 大部分都包含了操 作繁琐的 SPE 净化步骤, 成本较高。国家标准方 法 ${ }^{[16]}$ 称取 $5 \mathrm{~g}$ 牛奶, 最后定容至 $2 \mathrm{~mL}$, 浓缩了约
2.5 倍, SPE 柱净化后样品足够干净。本方法称取 1 $\mathrm{g}$ 牛奶, 最后定容至 $1 \mathrm{~mL}$, 正己烷除脂后, 采用空白 基质匹配标准溶液法 ${ }^{[17]}$ 进行校准, 检测结果显示样 品同样足够干净。而本试验无需 SPE 柱净化, 成本 较低, 简便易行, 效率高, 定量限为 $2 \mu \mathrm{g} / \mathrm{kg}$, 灵敏度 更高。

\subsection{2 提取液及浓缩条件的选择}

牛奶中蛋白质较多, 本试验比较了乙腈、甲醇和 乙醇沉淀蛋白的效果, 结果发现乙腈的沉淀效果更 好, 头孢洛宁的回收率较高, 在 75\% 以上; 甲醇和乙 醇沉淀蛋白效果不如乙腈, 且回收率也相对低些 (约 60\%) , 所以最后选用乙腈作为蛋白沉淀剂。考 察了沉淀 $1 \mathrm{~g}$ 牛奶中蛋白质所需要的乙腈的体积, 尝试过 4、5、6、8、10 $\mathrm{mL} 。 4 \mathrm{~mL}$ 时提取不充分, 回收 率约 65\%; $5 \mathrm{~mL}$ 时回收率为 75\% 85\% ; 连续增加 乙腈体积至 $10 \mathrm{~mL}$, 回收率几乎没有提高。为了节 约试剂及提高方法的简便性, 最终确定采用 $5 \mathrm{~mL}$ 乙腈进行提取。氮气流吹干浓缩时考察了温度分别 为 $30 、 37 、 40{ }^{\circ} \mathrm{C}$ 时对头狍洛宁的影响。结果表明 30 ${ }^{\circ} \mathrm{C}$ 下浓缩效率低, 单个样品吹干所需时间为 $1.2 \mathrm{~h}$, 回收率为 $74 \% \sim 80 \% ; 40{ }^{\circ} \mathrm{C}$ 下浓缩效率高, 但头孢 洛宁有所降解, 回收率只有 $58 \% \sim 65 \%$; 在 $37{ }^{\circ} \mathrm{C}$ 时浓 缩效率较高, 单个样品吹干所需时间为 $40 \mathrm{~min}$, 且 头孢洛宁降解不明显, 回收率为 $75 \% \sim 87 \%$ 。在浓缩 吹干后, 加人 $1 \mathrm{~mL}$ 甲醇- $0.1 \%$ 甲酸水溶液 $(3: 7, \mathrm{v} /$ $\mathrm{v}$ )复溶, 加人正己烷进一步除去脂溶性杂质, 低温 高速离心后过 $0.22 \mu \mathrm{m}$ 滤膜可保证样品足够干净。

\section{4 标准曲线和线性范围}

准确称取 $1 \mathrm{~g}$ 空白牛奶 7 份分别置于 $15 \mathrm{~mL}$ 离 心管中, 按 1.3 节方法处理样品, 用所得空白基质液 分别配制头孢洛宁标准工作液 $(2 、 5 、 10 、 20 、 50 、 100$ 、 $200 \mu \mathrm{g} / \mathrm{L}$ ), 用 HPLC-MS/MS 进行检测。每一水平 设 5 个平行样品同日检测, 连续测定 5 日。以头孢 洛宁峰面积平均值为纵坐标 $(Y)$, 质量浓度为横坐 标 $(X, \mu \mathrm{g} / \mathrm{L})$ 绘制标准曲线, 求得牛奶中头孢洛宁 的回归方程 $Y=2944.9 X+2227.5$, 相关系数 $r=$ 0.9996 。

\section{5 检出限和定量限}

准确称取空白牛奶 $1 \mathrm{~g}$, 依次加人不同质量浓度 $(1 、 5 、 10 、 20 、 50 \mu \mathrm{g} / \mathrm{L})$ 的头孢洛宁标准工作液 100 $\mu \mathrm{L}$, 浴旋混匀, 使得样品中头孢洛宁的添加浓度分 别为 $0.1 、 0.5 、 1 、 2 、 5 \mu \mathrm{g} / \mathrm{kg}$, 按 1.3 节方法处理样 品。以 $S / N \geqslant 3$ 确定检出限 (LOD), 以 $S / N \geqslant 10$ 确 定定量限 $(\mathrm{LOQ})$ 。牛奶中头孢洛宁的检出限为 0.5 $\mu \mathrm{g} / \mathrm{kg}$, 定量限为 $2 \mu \mathrm{g} / \mathrm{kg}$ 。 


\section{6 回收率和精密度的测定}

回收率和精密度采用在空白牛奶中添加标准溶 液的方法进行检测。其 MRM 谱图见图 3 和图 4。 根据残留限量要求及欧盟对头孢洛宁设置的 MRL, 在 LOQ、1/2MRL、MRL、2MRL 4 个添加水平, $1 \mathrm{~g}$ 空 白牛奶中分别添加 $20 、 50 、 100 、 200 \mu \mathrm{g} / \mathrm{L}$ 的头孢洛 宁标准工作液 $100 \mu \mathrm{L}$, 即牛奶中添加浓度为 $2 、 5$ 、 $10 、 20 \mu \mathrm{g} / \mathrm{kg}$, 旋浴混匀, 按 1.3 节方法处理样品, 每 一水平设 5 个平行样品同日检测, 连续测定 5 日。 求得空白牛奶中添加头孢洛宁回收率的平均值 $(X)$ 和标准差 $(\mathrm{SD})$, 同时计算日内和日间相对标准偏 差。结果表明,头孢洛宁在牛奶中的平均回收率介 于 78. 5\% 86. $2 \%$ 之间, 日内相对标准偏差为 $1.5 \%$ $\sim 6.2 \%$, 日间相对准偏差为 $2.9 \% \sim 5.6 \%$ （见表 1)。

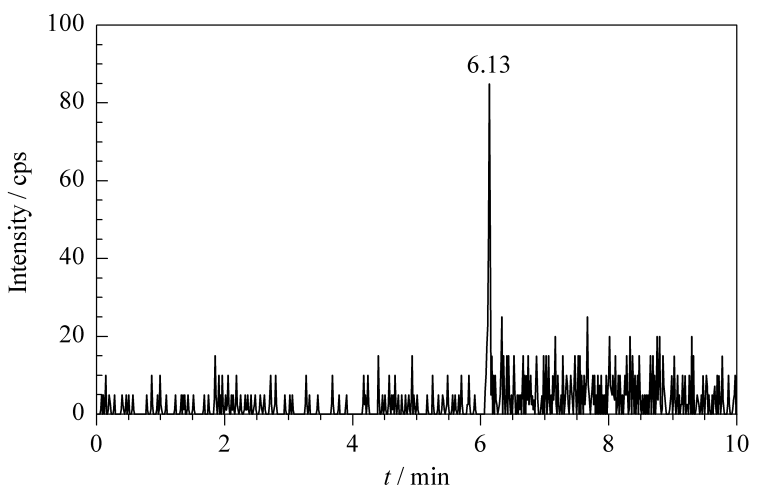

图 3 空白牛奶的 MRM 谱图

Fig. 3 MRM chromatogram of a blank milk sample

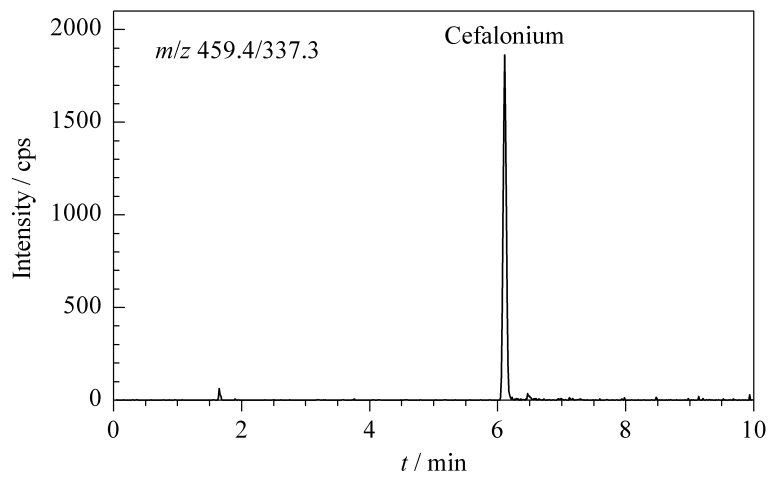

图 4 空白牛奶添加 $2 \mu \mathrm{g} / \mathrm{kg}$ 头孢洛宁的 MRM 谱图

Fig. 4 MRM chromatogram of a blank milk sample spiked with cefalonium at $2 \mu \mathrm{g} / \mathrm{kg}$

\section{7 稳定性}

取 20 份空白牛奶, 添加 MRL 水平的头孢洛宁 标准溶液, 按 1.3 节方法处理样品, 用 HPLC-MS/ MS 进行检测,记录其峰面积; 于室温条件下放置 24 $\mathrm{h}$ 后重新检测, 比较两次峰面积的变化。结果显示, 牛奶样品中头孢洛宁的峰面积变化在 $-3.35 \%$ $3.80 \%$ 之间,说明样品在室温条件下放置 $24 \mathrm{~h}$ 内，
表 1 牛奶中头孢洛宁的加标回收率及相对标准偏差 $(\boldsymbol{n}=\mathbf{5})$

Table 1 Recoveries and relative standard deviations ( RSDs ) of cefalonium spiked in milk $(n=5)$

\begin{tabular}{cccc}
\hline $\begin{array}{c}\text { Spiked/ } \\
(\mu \mathrm{g} / \mathrm{kg})\end{array}$ & $\begin{array}{c}\text { Recovery/ } \\
\%\end{array}$ & $\begin{array}{c}\text { Intra-day RSD/ } \\
\%\end{array}$ & $\begin{array}{c}\text { Inter-day RSD/ } \\
\%\end{array}$ \\
\hline 2 & $78.5 \pm 2.3$ & 1.8 & 2.9 \\
5 & $84.5 \pm 3.7$ & 2.3 & 4.4 \\
10 & $86.2 \pm 3.1$ & 1.5 & 3.6 \\
20 & $80.8 \pm 4.5$ & 6.2 & 5.6 \\
\hline
\end{tabular}

头孢洛宁稳定性良好。

\section{8 基质效应的消除}

样品基质溶液对待测物的电喷雾离子化影响较 大, 可抑制或增强信号响应, 导致检测结果的准确度 与精密度降低。本试验通过在空白牛奶基质提取液 中添加低、中、高 3 个浓度即 $2 、 20 、 200 \mu \mathrm{g} / \mathrm{L}$ 的头孢 洛宁标准溶液, 与纯溶剂中的信号强度进行比较, 牛 奶基质峰面积与纯溶剂的峰面积比值分别为 64\% $92 \% 、 75 \% \sim 88 \% 、 80 \% \sim 86 \%$, 说明牛奶基质对头孢洛 宁检测有抑制效应。为消除样品基质效应的影响, 采用空白基质匹配标准溶液法进行校准。

\section{9 抽样检测}

从不同市场购买新鲜牛奶 10 份, 从超市购买国 产蒙牛、伊利品牌盒装牛奶各 10 份, 以及新西兰、澳 大利亚进口盒装牛奶各 10 份, 每份 $100 \mathrm{~mL}$, 共 50 份样品, 按本方法进行样品前处理和检测。结果显 示,所有国产样品均未检测出头孢洛宁残留,有 1 份 新西兰进口样品检测出 $8.0 \mu \mathrm{g} / \mathrm{kg}$ 头狍洛宁残留。 这是因为头孢洛宁在国内尚未批准使用, 有关头孢 洛宁防治奶牛乳房炎的制剂还处于研发阶段,尚未 投人生产。上述结果表明该方法可用于检测牛奶中 头狍洛宁的残留量。

\section{3 结论}

本文建立了 HPLC-MS/MS 测定牛奶中头孢洛 宁残留量的分析方法。样品经乙腈提取, 氮气吹干 后用甲醇- $0.1 \%$ 甲酸水溶液 $(3: 7, \mathrm{v} / \mathrm{v})$ 复溶, 正己 烷去除脂肪后, 经 HPLC-MS/MS 分析。本方法前 处理操作简便易行,不需要 SPE 柱净化,所需试剂 较少,成本较低,且精密度和重复性好,灵敏度高, 定 量限低于欧盟对牛奶中头孢洛宁制定的最高残留限 量,适用于牛奶中头孢洛宁的残留检测。

\section{参考文献:}

[1] Cephalonium (头孢洛宁). [2013-12-16]. http://www. biomart.cn/infosupply/5329604.htm

[2] Bryan M A, Heuer C, Emslie F R. New Zeal Vet J, 2011, 59 (4) : 166 
[3] Shephard R W, Burman S, Marcun P. Aust Vet J, 2004, 82 (10): 624

[4] McDougall S. New Zeal Vet J, 2010, 58(1) : 45

[5] Bai G T, Chu X G, Pan G Q, et al. Chinese Journal of Chromatography (白国涛, 储晓刚, 潘国卿, 等. 色谱), 2009, 27 (4) : 417

[6] Cefalonium Summary Report (2). Committee for Veterinary Medicinal Products. [2013-12-25]. http://www.ema.europa. eu/docs/en/2009/11/WC500011741.pdf

[7] National Food Safety Resource Database (中国食品安全资 源数据库). (2007-06-28). http://www.fsr.org.cn/Index.asp

[8] Kantiani L, Farre M, Barcelo D. TrAC-Trend Anal Chem, 2009, 28(6): 729

[9] Beltrán M C, Romero T, Althaus R L, et al. J Dairy Sci, 2013, 96(5): 2737

[10] Bremus A, Dietrich R, Dettmar L, et al. Anal Bioanal Chem, 2012, 403(2): 503
[11] Hou X L, Wu Y L, Lv Y, et al. J Chromatogr B, 2013, 931 (15) : 6

[12] Becker M, Zittlau E, Petz M. Anal Chim Acta, 2004, 520 $(1 / 2): 19$

[13] Ye X M, Cao X J, Ma Y P, et al. Journal of Instrumental Analysis (叶学敏, 曹小吉, 马永萍, 等. 分析测试学报), $2010,29(12): 1135$

[14] Guo C N. [ MS Dissertation ]. Guangzhou: South China Agricultural University ( 郭春娜. [硕士学位论文]. 广州: 华 南农业大学), 2009

[15] Yang G, Huang X H, Guo C N, et al. Chinese Journal of Chromatography (杨刚, 黄显会, 郭春娜, 等. 色谱), 2012, $30(6): 568$

[16] GB/T 22989-2008

[17] He L M, Liu X G, Zeng Z L. Chinese Journal of Chromatography (贺利民, 刘祥国, 曾振灵. 色谱), 2008, 26(1) : 98 